\title{
The Posteroanterior Locking for the Distal Humerus Nailing. Is It a Viable Option?
}

\author{
Yogesh Salphale, Jagannath Kaginalkar, Wasudeo Mahadeo Gadegone, Kiran Janwe \\ Shushrusha Multispecialty Hospital, Chandrapur, India \\ Email: yosa@aol.in
}

How to cite this paper: Salphale, Y., Kaginalkar, J., Gadegone, W.M. and Janwe, K. (2017) The Posteroanterior Locking for the Distal Humerus Nailing. Is It a Viable Option? Surgical Science, 8, 488-492. https://doi.org/10.4236/ss.2017.811054

Received: October 26, 2017

Accepted: November 22, 2017

Published: November 27, 2017

Copyright $\odot 2017$ by authors and Scientific Research Publishing Inc. This work is licensed under the Creative Commons Attribution International License (CC BY 4.0).

http://creativecommons.org/licenses/by/4.0/

\section{(c) (i) Open Access}

\begin{abstract}
The distal interlocking is regarded as an inherent part of the antegrade humeral nailing technique. Traditionally the distal locking of intramedullary humeral nails is achieved using a freehand technique. The humerus nailing locking options in the conventional nailing systems are placed in the anterior-posterior and lateral-medial directions. It exposes both the patient and surgeon to radiation, is time consuming, and has a potential risk of damaging neurovascular structures, especially the radial and lateral cutaneous nerve when the anterior-posterior and lateral-medial locking constructs are used. We aim to present a technical tip to ease the distal locking procedure and avoid the possible neurovascular complications with the existing nailing systems.
\end{abstract}

\section{Keywords}

Humerus Fracture, Humerus Nailing, Posteroanterior Locking, Interlocking Nailing

\section{Technique}

A 28 year old male sustained a closed Humeral diaphyseal fracture and presented to our casualty. After having an informed consent and approval from the Hospital ethics committee regarding the use of the Intra operative pics and the pre and post operative Xrays (in Figure 1(a) and Figure 1(b)), nailing was performed with the patient in the supine position with the image intensifier at $90^{\circ}$ to the patient [Figure 2(a)]. After the insertion of the humerus nail, the proximal fragment is locked with the suitable number of the locking bolts as per the fracture configuration and the fracture fragments are closely approximated, the patient is then turned to the left Lateral decubitus position for the right humeral shaft and vice versa [Figure 2(b)]. The draping for the distal locking is adjusted 


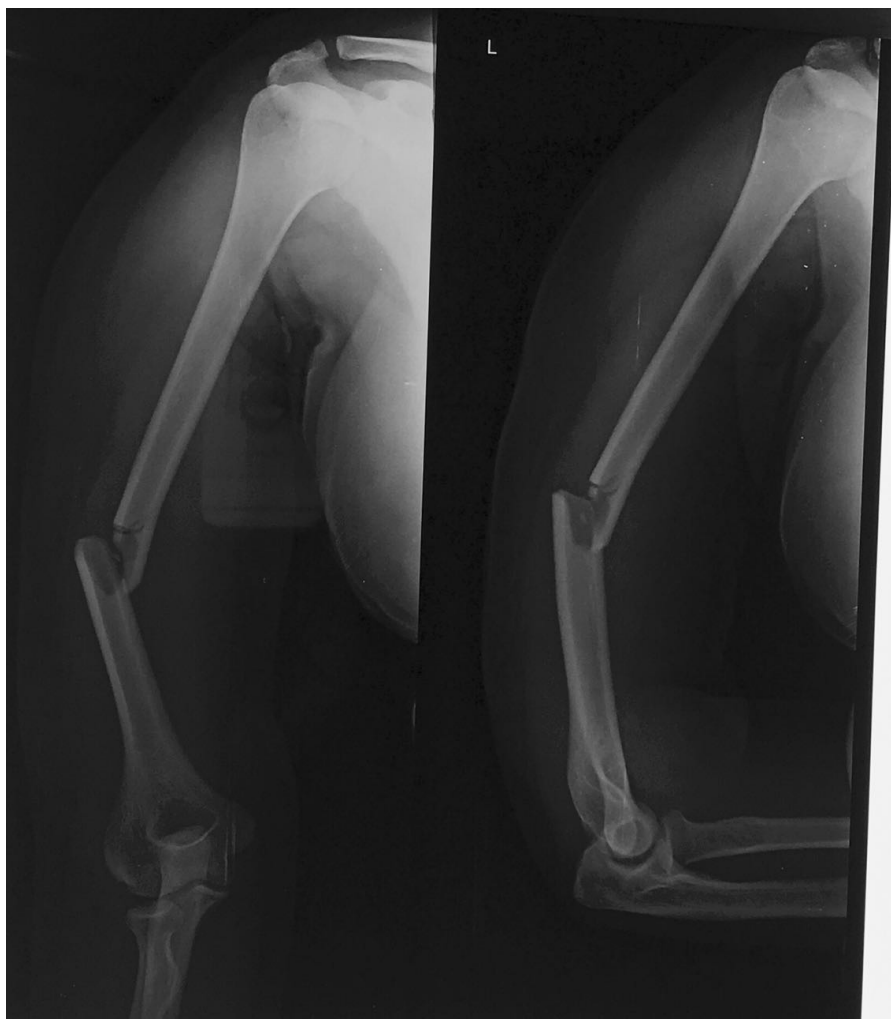

(a)

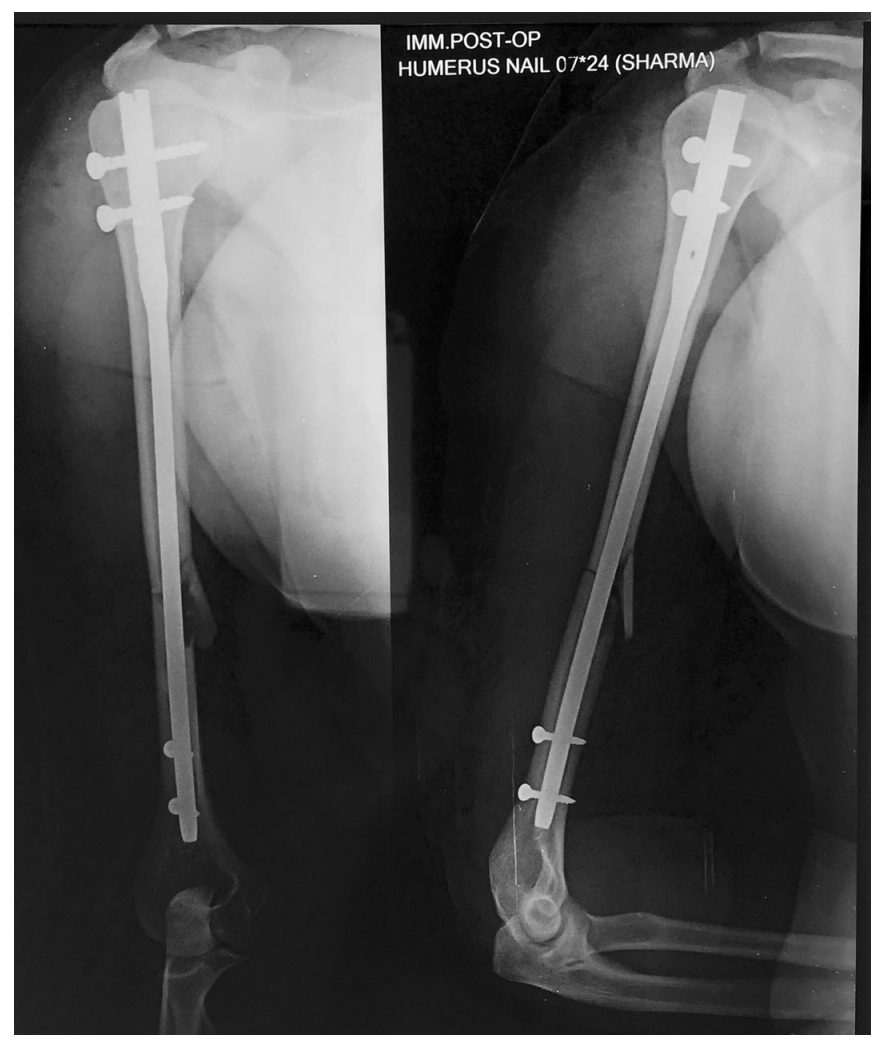

(b)

Figure 1. (a) Shows the Preoperative Xray; (b) postoperative X ray of the diaphyseal humerus fracture with PA locking screws. 


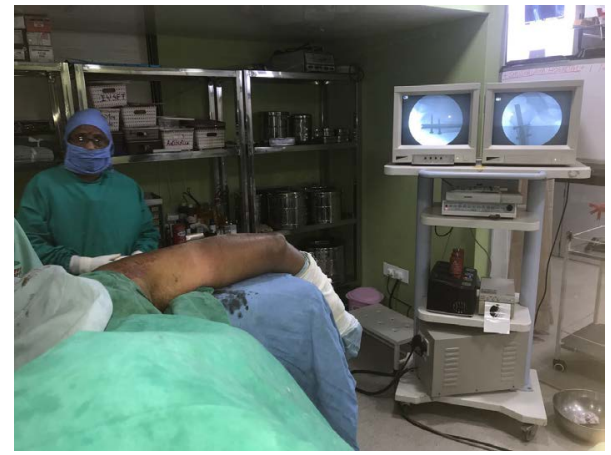

(a)

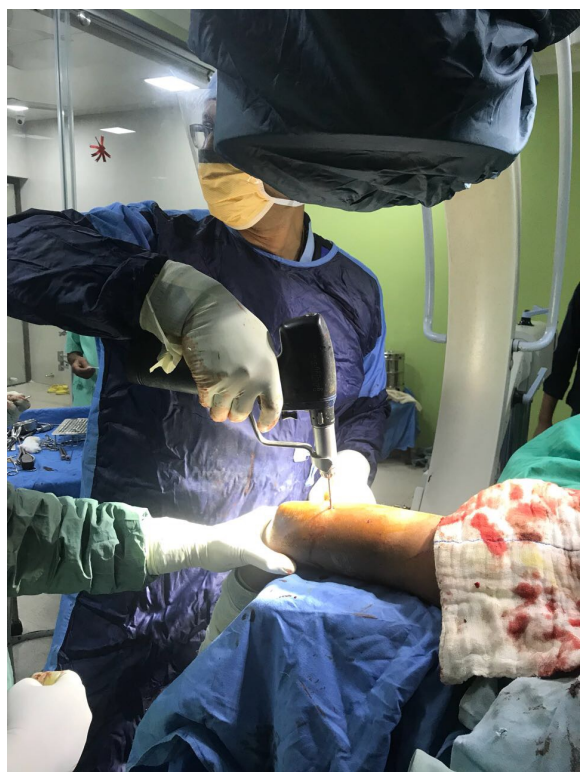

(b)

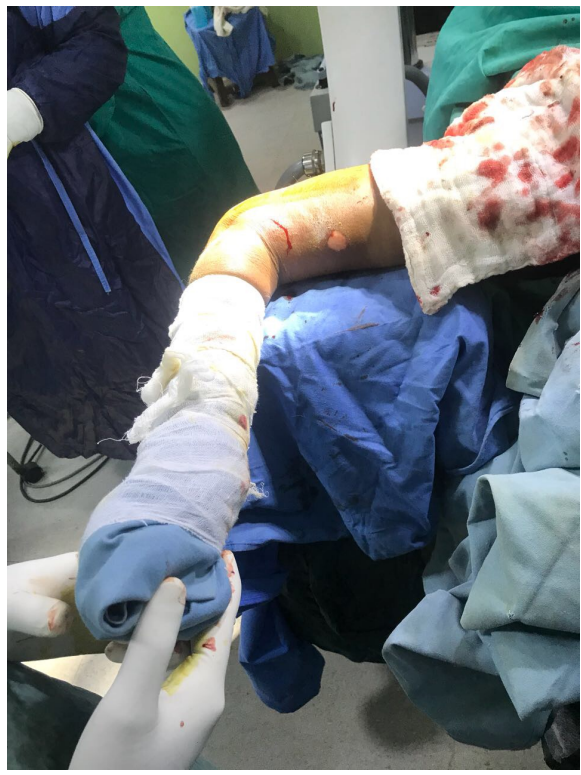

(c)

Figure 2. Showing the operative setup. (a) Showing the position of the patient; (b) showing the distal locking being carried; (c) arm rotated to visualise the lateral humerus. 
and the arm then rests on the arm board [Figure 2(c)]. The amount of padding, arm rotation can be adjusted to give a perfect view of the distal locking hole without requiring an assistant. In this position, we then perform distal locking in a posterior-anterior direction after locating the distal locking hole with the $\mathrm{C}$ arm.

\section{Discussion}

Proximal and distal locking are integral elements for rotational stability of an IMN [1] [2]. Yet distal locking is associated with some issues: Lateral view of the humerus is not easily obtained, locking holes are narrow, the lateral surface of the humerus is "slippery" due to the peculiar anatomy and the danger of injury to the radial nerve and other vulnerable structures always looms [3] [4] [5].

The options for the distal interlocking nails for the humerus nails may have either AP directed screws or medial-lateral directed screws to achieve fixation in the distal fragment. The distal part of the humerus is funnel shaped and the anterior cortical surface above the olecranon fossa is especially hard. Achieving a suitable entry point in the AP directed screws, the incision is made just lateral to the biceps tendon. It often entails the use of a small diameter Steinman pin or a $2 \mathrm{~mm} \mathrm{~K}$ wire before drilling to have a small footprint on the distal humerus before proceeding with the locking.

Whereas with the lateral to medial screw insertion the radial nerve was at direct risk. The ulnar nerve and median nerve/brachial artery bundle was at risk if significant overpenetration of the medial cortex occurred. The musculocutaneous nerve was at direct risk with placement of a distal screw from anterior to posterior nails [6].

Only one reference [7] has been mentioned in the literature regarding the posteroanterior locking. It involved turning the patients arm and placing it on the pelvic support board, which we found uncomfortable.

The advantage of the posteroanterior locking is the ease with which it could be performed. There are obviously no anatomical structures which are at risk. The amount of scar is minimal.

In the prone position, the visualization of the adequate area of the humeral shaft with the reduced fracture is possible. Any minor adjustments or changes are possible. The arm can be easily turned to ascertain the length of the distal screws after insertion.

We strongly feel the efforts to turn the patient to the desired position of posteroanterior locking and a minor change in the draping and the $\mathrm{C}$ arm position far outweighs the potential of minimising the complications associated with the anteriorly based neurovascular bundles.

As this procedure has not been described in the literature apart from the one mentioned, we strongly feel that the posteroanterior locking is a relatively easy step, which obviates the need of dissection of the structures on the anterior aspect of the distal humerus and which could be performed with minimal difficul- 
ty at any hospital.

\section{References}

[1] Tyllianakis, M., Tsoumpos, P., Anagnostou, K., Konstantopoulou, A. and Panagopoulos, A. (2013) Intramedullary Nailing of Humeral Diaphyseal Fractures. Is Distal Locking Really Necessary? International Journal of Shoulder Surgery, 7, 65-69. https://doi.org/10.4103/0973-6042.114233

[2] Moran, M.C. (1995) Distal Interlocking during Intramedullary Nailing of the Humerus. Clinical Orthopaedics and Related Research, No. 317, 215-218.

[3] Garnavos, C. (2005) Humeral Nails: When to Choose What and How to Use. Current Orthopaedics, 19, 294-304. https://doi.org/10.1016/j.cuor.2005.06.003

[4] Garnavos, C. (2011) Diaphyseal Humeral Fractures and Intramedullary Nailing: Can We Improve Outcomes? Indian Journal of Orthopaedics, 45, 208-215. https://doi.org/10.4103/0019-5413.67117

[5] Garnavos, C. (2001) Intramedullary Nailing for Humeral Shaft Fractures: The Misunderstood Poor Relative. Current Orthopaedics, 15, 68-75.

https://doi.org/10.1054/cuor.2001.0166

[6] Rupp, R.E., Chrissos, M.G. and Ebraheim, N.A. (1996) The Risk of Neurovascular Injury with Distal Locking Screws of Humeral Intramedullary Nails. Orthopedics, 19, 593-595.

[7] White, W.J., Hawken, R.M.A. and Giles, N.C. (2012) Posterior to Anterior Distal Locking of Humeral Intramedullary Nails. Annals of the Royal College of Surgeons of England, 94, 603. https://doi.org/10.1308/rcsann.2012.94.8.603 